\title{
Performance analysis of a low-complexity and efficient QoS differentiation algorithm for bufferless optical packet switches with shared wavelength converters in asynchronous operation
}

Nord, Martin

Published in:

First International Conference on Broadband Networks, 2004. BroadNets 2004. Proceedings.

Link to article, DOI:

10.1109/BROADNETS.2004.58

Publication date:

2004

Document Version

Publisher's PDF, also known as Version of record

Link back to DTU Orbit

Citation (APA):

Nord, M. (2004). Performance analysis of a low-complexity and efficient QoS differentiation algorithm for bufferless optical packet switches with shared wavelength converters in asynchronous operation. In First International Conference on Broadband Networks, 2004. BroadNets 2004. Proceedings. (pp. 334-336). IEEE. https://doi.org/10.1109/BROADNETS.2004.58

\section{General rights}

Copyright and moral rights for the publications made accessible in the public portal are retained by the authors and/or other copyright owners and it is a condition of accessing publications that users recognise and abide by the legal requirements associated with these rights.

- Users may download and print one copy of any publication from the public portal for the purpose of private study or research.

- You may not further distribute the material or use it for any profit-making activity or commercial gain

- You may freely distribute the URL identifying the publication in the public portal 


\title{
Performance Analysis of a Low-Complexity and Efficient QoS Differentiation Algorithm for Bufferless Optical Packet Switches with Shared Wavelength Converters in Asynchronous Operation
}

\author{
M. Nord \\ Research Centre COM, Technical University of Denmark, B345V, Lyngby, Denmark \& \\ Telenor R\&D, 1331 Fornebu, Norway. \\ mn@com.dtu.dk
}

\begin{abstract}
This paper presents a simulation study of a lowcomplexity optical packet switching Quality of Service differentiation scheme, aiming at minimising the penalty of offering packet loss rate isolation in an optical packet switch with a wavelength converter pool. Special emphasis is given to potential improvements, impact of node dimensions and overload situations.
\end{abstract}

\section{Introduction}

Efficient contention resolution, avoiding excessive hardware counts, is an important target in Optical Packet Switching (OPS) and Optical Burst Switching (OBS) research. This has motivated Tuneable Wavelength Converter (TWC) pool designs, e.g. Shared Per Node (SPN) [1-2], and Shared Per Waveband Plane (SPWP) [3]. Moreover, asynchronous operation with variable length packets is attractive, avoiding complex synchronisers and providing a good match with Internet traffic [4]. Finally, differentiating Quality of Service (QoS) in the optical layer may facilitate the transition from a Best-Effort (BE) to a QoS aware Internet [4]. This paper addresses these issues, by investigating the performance of a recently proposed QoS differentiation algorithm, suitable for an SPN optical packet switch [5].

\section{Best-Effort switch performance}

We evaluate performance of a single optical packet switch by discrete event-driven numerical simulations in OPNET. Asynchronous operation is implemented by a perwavelength Poisson packet arrival process with exponential packet length distribution, subject to per-wavelength FIFO buffers to emulate serialised packet output clocking. Packet $\mathrm{CoS}$ and output fibre are uniformly distributed. The generic node design is illustrated in Figure 1, where the Wavelength Conversion Ratio (WCR) denotes the relative size of the SPN TWC pool, thus governing TWC- and switch matrix port count. In a BE scenario, Figure 2 shows the impact of the node adjacency, F, the wavelength count on each fibre, $\mathrm{W}$, and the WCR, with $95 \%$ confidence intervals. For a fixed load, $\mathrm{L}=0.7$, the PLR decreases down to a minimum, given by $\mathrm{F}$ and $\mathrm{W}$, with increasing WCR. Hence, achievable TWC count reduction depends on the required PLR.

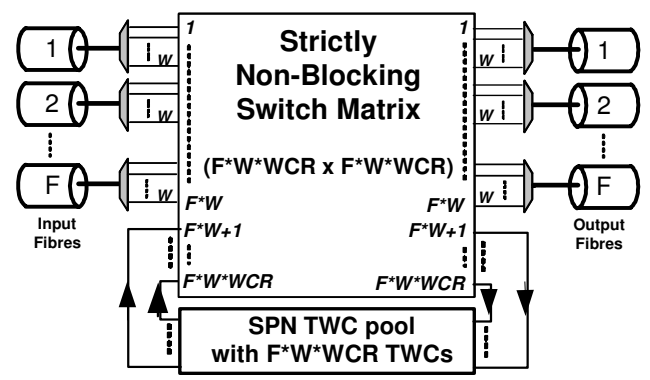

Figure 1. SPN switch with TWC pool

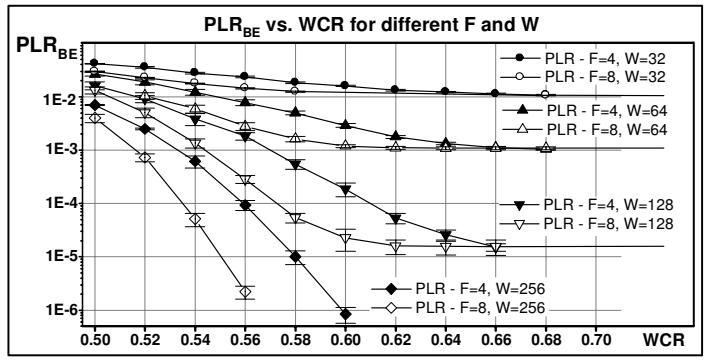

Figure 2. BE performance.

\section{QoS differentiation algorithm}

When the traffic consists of two Classes of Service $(\mathrm{CoS})$ with different PLR requirements, QoS differentiation has two potential benefits: i) achieve lower minimum PLR than 
imposed by L, F and $\mathrm{W}$ in the BE case, and ii) operate with a lower WCR than the BE switch, whilst still comply with the most demanding $\mathrm{CoS}$.

Figure 3 details the QoS algorithm, aiming at obtaining a good performance-complexity trade-off, by using an Access Restriction (AR) based approach. A TWC is only used if the packet cannot achieve Direct Mapping (DM). In this case, the scheduler only allocates $\operatorname{CoS} 2$ packets if the number of free output wavelengths (OWLs) on the requested output fibre, $F$, and the number of free TWCs in the pool, termed $\mathrm{N}_{\text {OWL }}(\mathrm{F})$ and $\mathrm{N}_{\text {TWC }}$ respectively, are above a threshold defined by the ratios $\mathrm{R}_{\mathrm{WL}}$ and $\mathrm{R}_{\mathrm{WC}}$, respectively. Ideally, any QoS motivated discard of the $\operatorname{CoS} 2$ packet should be rewarded by avoidance of loss of a CoS1 packet. However, in asynchronous operation, the scheduling is done without knowing its effects on later arriving packets. An optimum AR threshold choice has a minimum increase in total PLR compared to the BE switch, thus minimum PLR PENALTY, for the desired difference in the PLR isolation ratio of the two

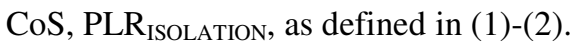

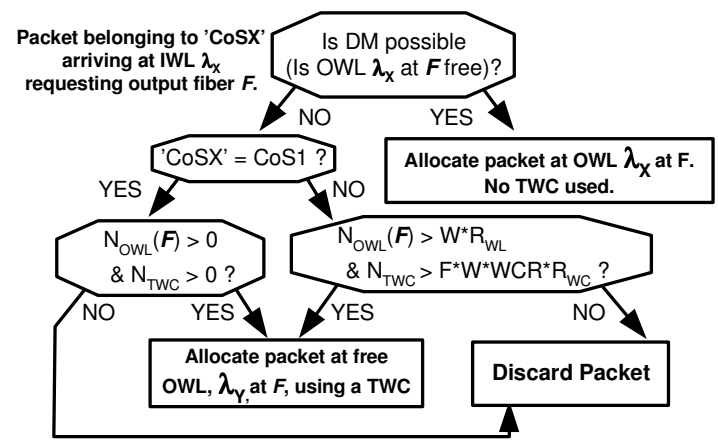

Figure 3. QoS algorithm

$$
\begin{aligned}
& P L R_{\text {ISOLATION }}=\frac{P L R_{\text {CoS } 2}}{P L R_{\text {COS } 1}} \\
& P L R_{P E N A L T Y}=\frac{P L R_{\text {With_QoS_Diff }}}{P L R_{B E}}=\frac{0.5\left(P L R_{C O S 1}+P L R_{\text {CoS2 } 2}\right)}{P L R_{B E}}
\end{aligned}
$$

\section{QoS algorithm performance}

\subsection{Single- vs. two dimensional AR schemes}

Figure 4 illustrates the PLR of $\operatorname{CoS} 1$ and of $\operatorname{CoS} 2$ as a function of the AR parameters, by scanning five $\mathrm{R}_{\mathrm{WC}}$ values for each of the three $R_{W L}$ values, for $\mathrm{W}=64$ and for $\mathrm{W}=128$. The WCR values are 0.625 and 0.57 , respectively. In both cases, when both $\mathrm{R}_{\mathrm{WC}}$ and $\mathrm{R}_{\mathrm{WL}}$ are zero, PLR $\mathrm{R}_{\mathrm{CoS} 1}$ and PLR $_{\mathrm{CoS} 2}$ are approximately equal, and the resulting PLR $\mathrm{BE}_{\mathrm{BE}}$ values of $(1.6 \pm 0.1) \times 10^{-3}$ and $(1.1 \pm 0.1) \times 10^{-3}$, respectively, match well corresponding curves in Figure 2.

Figure 5 illustrates resulting penalty as a function of isolation. When both $\mathrm{R}_{\mathrm{WC}}$ and $\mathrm{R}_{\mathrm{WL}}$ are zero, the isolation is 1. As shown in [5], the two-dimensional approach with AR on both TWCs and OWLs, can obtain lower penalties than the one-dimensional "pure WL reservation" and "pure WC reservation" approaches.

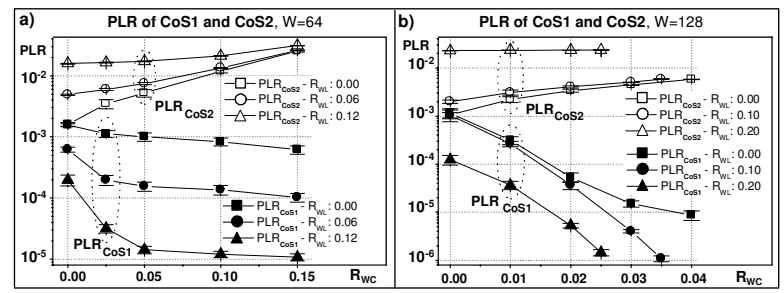

Figure 4. QoS algorithm performance, PLR.

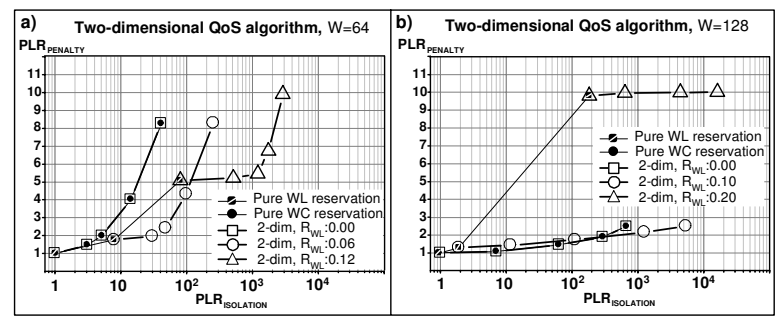

Figure 5. QoS algorithm performance, penalty.

\subsection{Impact of Direct Mapping}

A peculiarity of the algorithm is that it allows CoS2 packets to be allocated even when either of the AR thresholds is violated, provided that the packet can find its own wavelength free at the requested output fibre. This Direct Mapping (DM) preference is introduced in order to save TWCs. Figure 6 shows that the DM preference does reduce penalty for the node with $\mathrm{W}=128$, but its impact depends on the desired isolation for $\mathrm{W}=64$.

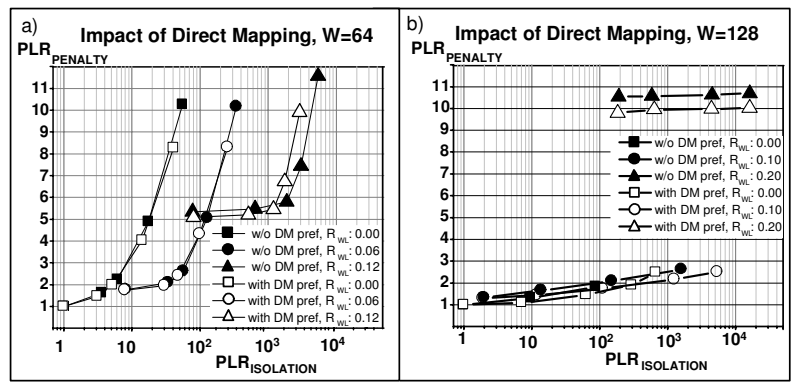

Figure 6. Impact of Direct Mapping

\subsection{Impact of Node Adjacency}

Figure 2 shows that increasing $\mathrm{F}$ from 4 to 8 , improves TWC sharing, and enables a lower WCR for the same $\mathrm{PLR}_{\mathrm{BE}}$. WCRs of 0.57 and 0.54 were chosen for $\mathrm{W}=64$ and $\mathrm{W}=128$, enabling $\mathrm{PLR}_{\mathrm{BE}}$ of $(2.8 \pm 0.4) \times 10^{-3}$ and $(1.4 \pm$ $0.1) \times 10^{-3}$, respectively. Figure 7 shows that, in spite of the reduced WCR, increasing $\mathrm{F}$ lowers the penalty; otherwise the behaviour of the QoS algorithm is quite similar. 


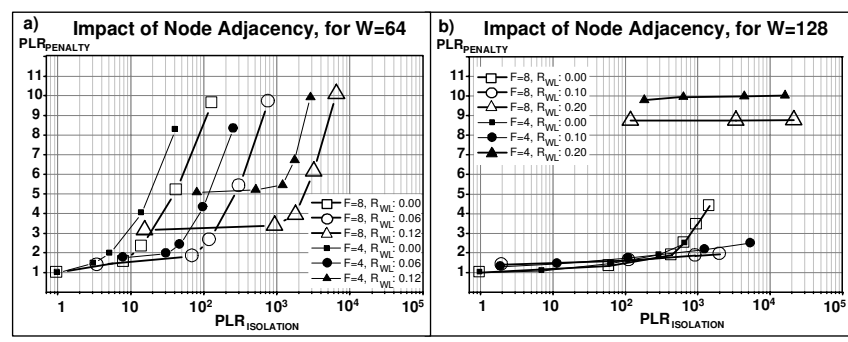

Figure 7. Impact of node adjacency.

\subsection{Impact of overload situations}

For statistical input traffic, the instantaneous input load at an optical packet switch fluctuates around the average value during simulations. In a real network, in periods of increased client layer traffic, the average load of the optical network may increase, unless a strict access policy is applied. Even so, it may also be that certain nodes experience higher average load than the network as a whole, in periods where unusually large portions of traffic passes by these nodes. On a BE network, increased network load increases PLR, as can be seen in Figure 8, for an isolation degree of 1 . After this initial penalty, the overload situation has a limited impact on the QoS algorithm differentiation so that the PLR of CoS1 can be kept below $10^{-5}$, by adjusting the AR parameters.
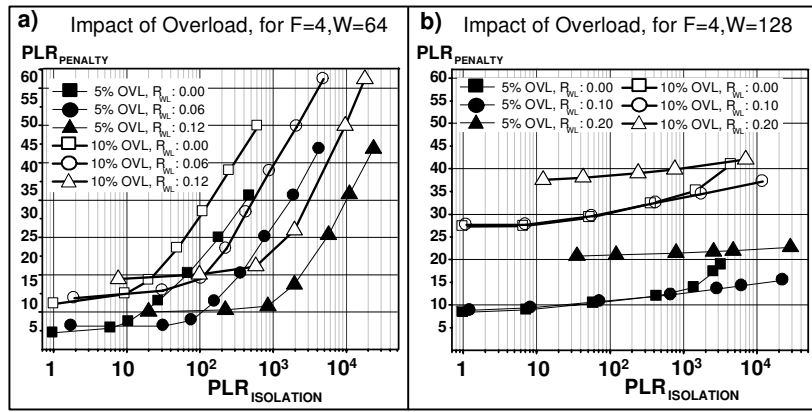

Figure 8. Impact of 5 and $10 \%$ overload.

\section{Discussion}

The granularity in the parameter scan was somewhat limited, and we expect increased granularity to demonstrate a slight performance improvement of the QoS differentiation schemes. Nevertheless, the study clearly shows that the two-dimensional QoS differentiation scheme outperforms single-dimensional systems.

Whether DM is beneficial or not was found to depend on the system parameters and the desired isolation ratio; hence, this issue should be studied in each case. It is expected that DM is more favourable when TWCs are scarce, and when the node adjacency is low, since DM packets also increases the probability of DM for later arriving packets that should be switched between the same fibres, thereby saving even more TWCs.

It was shown that performance improved when increasing $\mathrm{F}$ and $\mathrm{W}$, when maintaining or even lowering the WCR. However, hardware realisation issues limit scalability, such as limited switch matrix port counts and/or TWC wavelength operation range.

Considering load variations, decreases in load would lead to decreased penalties, particularly when the parameters of the QoS differentiation scheme are optimised, idem to overload situations. Hence, to make the QoS differentiation scheme as efficient as possible, the QoS scheme requires either capability of signalling such changes of load by the management system, or distributed load monitoring with associated parameter adjustment.

The choice of Poisson arrival will for most systems yield a better performance than more bursty traffic patterns [6]. On the other hand, high priority packets constitute as much as $50 \%$ of overall load. Lowering this ratio would significantly relax system requirements, enabling lower overall PLR and/or hardware savings in terms of WCR.

\section{Conclusion}

The AR-based QoS differentiation scheme is suitable for a SPN based TWC pool design. It is scalable with respect to node dimensions, and can maintain the PLR of the highpriority CoS, at the expense of an increase in the PLR of the low-priority $\mathrm{CoS}$, during overload situations.

\section{REFERENCES}

[1] V. Eramo, M. Listanti. "Packet Loss in a Bufferless Optical WDM Switch Employing Shared Tunable Wavelength Converters", IEEE Journ. Lightwave Techn., 18(12), pp. 18181833, December 2000.

[2] C. M. Gauger. "Performance of converter pools for contention resolution in optical burst switching", in Proc. $3^{\text {rd }}$ International Conference on Optical Networking and Communications (OptiComm 2002), July 2002, Boston, USA.

[3] M. Nord. "Waveband based multi-plane optical packet switch with partially shared wavelength converters", in Proc. $8^{\text {th }}$ IFIP Working Conference on Optical Networking Design and Modelling (ONDM 2004), Feb. 2004, Ghent, Belgium, pp. 179-198.

[4] F. Callegati et al. "Exploitation of DWDM for Optical Packet Switching With Quality of Service Guarantees", IEEE Journ. of Selected Areas in Communications, 20 (1), pp. 190-201, January 2002.

[5] M. Nord. "An efficient Quality of Service differentiation algorithm for buffer-less optical packet switches with partially shared wavelength converters", in Proc. of 3 rd International Conference on Optical Internet (OECC-COIN 2004), July 2004, Yokohama, Japan, pp. 586-587.

[6] H. Overby, N. Stol. "Effects of bursty traffic in service differentiated Optical Packet Switched networks", OSA Optics Express, 12(3), pp. 410-415, February 2004. 